University of Wollongong

Research Online

Faculty of Engineering and Information

Faculty of Engineering and Information

Sciences - Papers: Part A

Sciences

2017

Correction factors to convert microdosimetry measurements in silicon to tissue in ${ }^{12} \mathrm{C}$ ion therapy

David Bolst

University of Wollongong, db001@uowmail.edu.au

Susanna Guatelli

University of Wollongong, susanna@uow.edu.au

Linh T. Tran

University of Wollongong

Lachlan Chartier

University of Wollongong, Ic752@uowmail.edu.au

Michael L. F Lerch

University of Wollongong, mlerch@uow.edu.au

See next page for additional authors

Follow this and additional works at: https://ro.uow.edu.au/eispapers

Part of the Engineering Commons, and the Science and Technology Studies Commons

Research Online is the open access institutional repository for the University of Wollongong. For further information contact the UOW Library: research-pubs@uow.edu.au 


\title{
Correction factors to convert microdosimetry measurements in silicon to tissue
} in ${ }^{12} \mathrm{C}$ ion therapy

\author{
Abstract \\ Silicon microdosimetry is a promising technology for heavy ion therapy (HIT) quality assurance, because \\ of its sub-mm spatial resolution and capability to determine radiation effects at a cellular level in a mixed \\ radiation field. A drawback of silicon is not being tissue-equivalent, thus the need to convert the detector \\ response obtained in silicon to tissue. This paper presents a method for converting silicon \\ microdosimetric spectra to tissue for a therapeutic ${ }^{12} \mathrm{C}$ beam, based on Monte Carlo simulations. The \\ energy deposition spectra in a $10 \mu \mathrm{m}$ sized silicon cylindrical sensitive volume (SV) were found to be \\ equivalent to those measured in a tissue SV, with the same shape, but with dimensions scaled by a factor \\ $K$ equal to 0.57 and 0.54 for muscle and water, respectively. A low energy correction factor was \\ determined to account for the enhanced response in silicon at low energy depositions, produced by \\ electrons. The concept of the mean path length (/Path) to calculate the lineal energy was introduced as an \\ alternative to the mean chord length (I) because it was found that adopting Cauchy's formula for the (I) \\ was not appropriate for the radiation field typical of HIT as it is very directional (/Path) can be determined \\ based on the peak of the lineal energy distribution produced by the incident carbon beam. Furthermore it \\ was demonstrated that the thickness of the SV along the direction of the incident ${ }^{12} \mathrm{C}$ ion beam can be \\ adopted as (IPath). The tissue equivalence conversion method and (IPath) were adopted to determine the \\ $\mathrm{RBE}_{10}$, calculated using a modified microdosimetric kinetic model, applied to the microdosimetric spectra \\ resulting from the simulation study. Comparison of the RBE10 along the Bragg peak to experimental TEPC \\ measurements at HIMAC, NIRS, showed good agreement. Such agreement demonstrates the validity of \\ the developed tissue equivalence correction factors and of the determination of ( $/$ Path).

\section{Disciplines} \\ Engineering | Science and Technology Studies

\section{Publication Details} \\ Bolst, D., Guatelli, S., Tran, L. T., Chartier, L., Lerch, M. L. F., Matsufuji, N. \& Rosenfeld, A. B. (2017). \\ Correction factors to convert microdosimetry measurements in silicon to tissue in ${ }^{12} \mathrm{C}$ ion therapy. \\ Physics in Medicine and Biology, 62 (6), 2055-2069.
}

\section{Authors}

David Bolst, Susanna Guatelli, Linh T. Tran, Lachlan Chartier, Michael L. F Lerch, Naruhiro Matsufuji, and Anatoly B. Rosenfeld 


\title{
Correction factors to convert microdosimetry measurements in silicon to tissue in ${ }^{12} \mathrm{C}$ ion therapy
}

\author{
David Bolst ${ }^{1}$, Susanna Guatelli ${ }^{1}$, Linh T. Tran ${ }^{1}$, Lachlan Chartier ${ }^{1}$, \\ Michael L. F. Lerch ${ }^{1}$, Naruhiro Matsufuji ${ }^{2}$, and Anatoly B. \\ Rosenfeld $^{1}$ \\ ${ }^{1}$ Centre for Medical Radiation Physics, University of Wollongong, Australia \\ ${ }^{2}$ Research Centre for Charge Particle Therapy, National Institute of Radiological Science, \\ Chiba, Japan \\ E-mail: db001@uowmail.edu.au
}

\begin{abstract}
Silicon microdosimetry is a promising technology for Heavy Ion Therapy (HIT) Quality Assurance, because of its sub-mm spatial resolution and capability to determine radiation effects at a cellular level in a mixed radiation field. A drawback of silicon is not being tissue-equivalent, thus the need to convert the detector response obtained in silicon to tissue. This paper presents a method for converting silicon microdosimetric spectra to tissue for a therapeutic ${ }^{12} \mathrm{C}$ beam, based on Monte Carlo simulations. The energy deposition spectra in a $10 \mu \mathrm{m}$ sized silicon cylindrical sensitive volume (SV) were found to be equivalent to those measured in a tissue SV, with the same shape, but with dimensions scaled by a factor $\kappa$ equal to 0.57 and 0.54 for muscle and water, respectively. A Low Energy Correction factor was determined to account for the enhanced response in silicon at low energy depositions, produced by electrons. The concept of the mean path length $\left\langle l_{P a t h}\right\rangle$ to calculate the lineal energy was introduced as an alternative to the mean chord length $\langle l\rangle$ because it was found that adopting Cauchy's formula for the $\langle l\rangle$ was not appropriate for the radiation field typical of HIT as it is very directional. $\left\langle l_{\text {Path }}\right\rangle$ can be determined based on the peak of the lineal energy distribution produced by the incident carbon beam. Furthermore it was demonstrated that the thickness of the SV along the direction of the incident ${ }^{12} \mathrm{C}$ ion beam can be adopted as $\left\langle l_{\text {Path }}\right\rangle$. The tissue equivalence conversion method and $\left\langle l_{P a t h}\right\rangle$ were adopted to determine the $\mathrm{RBE}_{10}$, calculated using a modified Microdosimetric Kinetic Model, applied to the microdosimetric spectra resulting from the simulation study. Comparison of the RBE 10 along the Bragg Peak to experimental TEPC measurements at HIMAC, NIRS, showed good agreement. Such agreement demonstrates the validity of the developed tissue equivalence correction factors and of the determination of $\left\langle l_{\text {Path }}\right\rangle$.
\end{abstract}

Keywords: Heavy ion therapy, Microdosimetry, Geant4

\section{Introduction}

Carbon therapy is a relatively new radiation treatment modality with first clinical trials starting in 1994 at the National Institute of Radiological Science (NIRS) in Japan [1]. One of the many advantages that ${ }^{12} \mathrm{C}$ therapy offers over conventional photon radiotherapy is its dose sparing to healthy tissue in treatment of deep-seated tumours, added to a higher radiobiological effectiveness (RBE). An underlying complication when delivering ${ }^{12} \mathrm{C}$ is that the $\mathrm{RBE}$ varies 
greatly, especially at the distal edge of the Bragg Peak (BP)/Spread Out Bragg Peak (SOBP) due to the mixed radiation field. As a result, an accurate RBE determination is crucial for the treatment planning and for routine Quality Assurance (QA).

The method adopted at the Heavy Ion Medical Accelerator in Chiba (HIMAC), NIRS, to calculate $\mathrm{RBE}_{10}$ values is by using the Microdosimetric Kinetic Model (MKM), formulated by Hawkins [2] [3], applied on microdosimetric measurements [4].

Microdosimetry is the study of the stochastic energy deposition in micron sized sensitive volumes (SVs) of a similar size to biological cells. The lineal energy $y$ is the energy $E$ deposited in an event divided by the average chord length $\langle l\rangle$. The lineal energy spectrum is described as $f(y)$; the first and second moment of $f(y)$ are $y_{F}$ and $y_{D}$, respectively. Experimentally, microdosimetry is performed by measuring the spectrum of deposited energy in the SV using a multi-channel analyser (MCA). The $f(y)$ spectrum is obtained by dividing the MCA spectrum by the $\langle l\rangle$.

Tissue Equivalent Proportional Counters (TEPCs) are the gold standard in microdosimetry, able to mimic a micron sized volume using tissue equivalent gas. They often adopt a spherical SV filled with a tissue equivalent gas (usually methane or propane based) operated at low pressures. When scaled by the density, the SV corresponds to a micron sized tissue equivalent SV. However TEPCs have many drawbacks including wall effects, operation complexity and poor spatial resolution [5] which are not ideal for the sharp dose profiles at the distal edge of the $\mathrm{BP} / \mathrm{SOBP}$ associated with carbon therapy.

As an alternative solution the Centre for Medical Radiation Physics (CMRP), University of Wollongong, has adopted a solid state approach which addresses the limitations of the TEPC. However silicon microdosimeters are not without their shortcomings, namely a lack of tissue equivalence.

Previous simulation studies have been performed to develop a method to convert microdosimetric spectra from silicon to tissue in a radiation field typical of space missions [6] and aviation [7]. This work addresses the development of a method to convert the microdosimetric spectra obtained in silicon to tissue for Heavy Ion Therapy (HIT). The fourth generation silicon microdosimeter designed by CMRP is refereed to as a "Mushroom" design and has been considered in this work.

In the microdosimetric approach, the mean chord length $\langle l\rangle$ is the average of randomly orientated chords in the volume of interaction [8]. In terms of particle tracks this means that the particle does not have any physical interaction in the volume. Currently Cauchy's formula (1) is usually adopted to determine the mean chord length $\langle l\rangle$, where $V$ and $S$ are the volume and surface area of the convex body:

$$
\left\langle l_{\text {Cauchy }}\right\rangle=\frac{4 V}{S}
$$

Equation (1) applies only for isotropic radiation fields by definition. In HIT the ${ }^{12} \mathrm{C}$ beam is incident normally on the detector and produces a secondary radiation field with different degrees of directionality depending on the type of nuclear recoil. This, together with the limitation of silicon fabrication of not being able to produce spherical SVs, may invalidate the use of Cauchy's formula for the determination of the average chord length. Based on these considerations, the second goal of this work is to investigate the appropriateness of Cauchy's formula for the microdosimeter in a typical HIT radiation field and eventually to propose a more suitable method.

\section{Method}

An ad-hoc Geant4 [9] [10] [11] simulation was developed to investigate the tissue equivalence of the Mushroom microdosimeter design and its chord length distribution in the HIT radiation 
field. Geant4 version 10.0 was adopted.

\subsection{The Geant4 simulation application}

Figure 1 (a) shows the set-up of the simulation. A mono-energetic $290 \mathrm{MeV} / \mathrm{u}{ }^{12} \mathrm{C}$ ion parallel beam, $5 \times 5 \mathrm{~mm}^{2}$ in size, is normally incident on a $30 \times 30 \times 30 \mathrm{~cm}^{3}$ water phantom with $4 \mathrm{~cm}$ of air between the primary beam position and the side of the phantom. The size of the beam was chosen because it is similar to the Pencil Scanning Beam voxel dimension adopted in Treatment Planning Systems [12]. The microdosimeter is placed in the centre of the beam and set at depths of $50 \mathrm{~mm}, 130 \mathrm{~mm}$ and $160 \mathrm{~mm}$ in the water phantom, corresponding to upstream, midstream and Bragg Peak positions along the Bragg curve. Such positions correspond to an average kinetic energy of the incident beam of, approximately, $232 \mathrm{MeV} / \mathrm{u}, 111 \mathrm{MeV} / \mathrm{u}$ and $9 \mathrm{MeV} / \mathrm{u}$, respectively, when reaching the Mushroom detector.

(a)

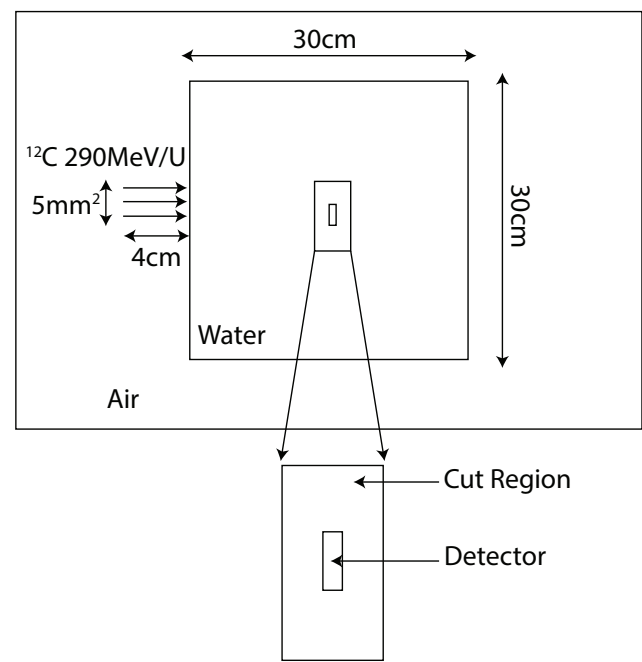

(b)

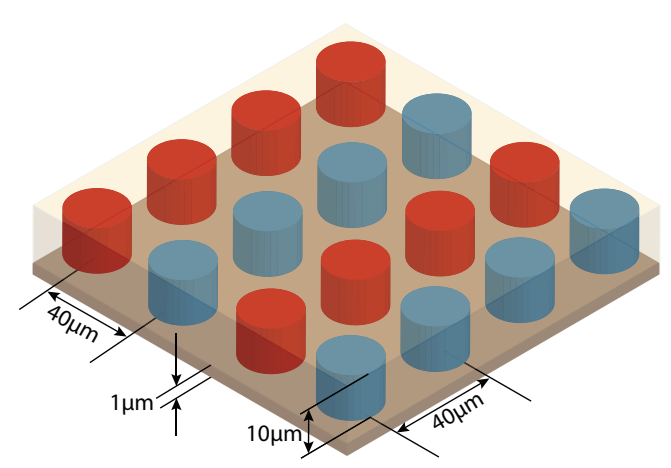

Figure 1: (a) Setup of the Geant4 simulation used for this study. (b) Diagram of the Mushroom microdosimeter. The blue and the red SVs represent the two different channels, over which the signal is integrated.

The Mushroom design features a fully 3D design with cylindrical silicon $n^{+}$and $p^{+}$electrodes with a height and diameter of $10 \mu \mathrm{m}$ and a pitch of $40 \mu \mathrm{m}$ between the centre of electrodes and being embedded in PMMA as shown in figure 1 (b). There are a total of 2500 sensitive volumes over an area of $2 \times 2 \mathrm{~mm}^{2}$, these SVs are divided into even and odd channels (shown as red and blue in the diagram) to reduce capacitance and co-incident events with each channel being summed into an individual preamplifier.

The electromagnetic interactions were modelled using G4 Standard EM option 3. G4HadronPhysicsQGSP_BIC_HP and G4HadronElasticPhysicsHP were used to describe the inelastic and elastic scattering of hadrons, respectively. The G4IonBinaryCascadePhysics is used to describe hadronic interactions of ions. For neutron interactions up to $20 \mathrm{MeV}$ the neutron High Precision (HP) model was used.

One event in the simulation includes the tracking of one primary carbon ion and its associated secondary particles. The readout of the detector was done by scoring the energy deposited in each channel over one event. The energy deposited per event is then integrated over the even and odd channels, separately, to mimic the readout of the real microdosimeter device.

In order to optimise the simulation execution time without sacrificing the accuracy of the results deriving from it, the Geant4 CutsPerRegion functionality was used. This strategy 
was chosen to avoid the tracking of secondary electrons which cannot reach the detector and deposit energy in it. Therefore the maximum kinetic energy of electrons generated by an incident $290 \mathrm{MeV} / \mathrm{u}{ }^{12} \mathrm{C}$ ion beam was estimated and their CSDA range $(R *)$ retrieved from the NIST ESTAR database [13]. The detector was positioned in the centre of a region, called a DetectorRegion, with sizes slightly exceeding $2 R *$. The threshold for production of secondary particles was fixed to $1 \mu \mathrm{m}$ in the Detector Region, and $R *$ outside.

\subsection{Determination of Tissue Equivalence for Heavy Ion Therapy}

The Geant4 simulation described in section 2.1 was adopted to determine the tissue equivalence of the Mushroom microdosimeter.

The method adopted follows the same methodology outlined by Guatelli et al. [6] and Davis et al. [14]. The energy deposition was calculated in the silicon SV $\left(\mathrm{SV}_{S i}\right)$ exposed to the HIT radiation field, along the Bragg curve, by means of Geant4. The $\mathrm{SV}_{S i}$ is a cylinder with $10 \mu \mathrm{m}$ diameter and height $\left(l_{S i}=10 \mu \mathrm{m}\right)$, corresponding to the actual dimensions of the device. Then the energy deposition distribution was calculated again, in the same radiation field conditions, with water and, alternatively, striated muscle, filling the SV. Water is considered as it is usually used as a good approximation of human tissue in radiotherapy Quality Assurance. The sizes of the water/muscle SV were then varied to obtain the same energy deposition spectrum as the one obtained in $\mathrm{SV}_{S i}$. The best agreement between the response in $\mathrm{SV}_{S i}$ and $\mathrm{SV}_{\text {water/muscle }}$ was quantified by comparing the energy deposition spectra in silicon and water/muscle by means of the $\chi^{2}$ test. A p-value larger than the significance level $\alpha(0.05)$ means that the two distributions do not vary significantly. The tissue equivalence correction factor $\kappa$ was then defined as the ratio of $l_{S i}$ to $l_{\text {water/muscle }}$ for which the best equivalent response to silicon was obtained.

\subsection{Path Length Distribution}

To investigate the appropriateness of the mean chord length provided by the Cauchy formula the mean path length, $\left\langle l_{\text {Path }}\right\rangle$, was introduced. Instead of being the mean of randomly distributed chords within the SV the $\left\langle l_{\text {Path }}\right\rangle$ is the mean of the path lengths of charged particles which cross the SV in the radiation field of interest. This allows to consider the directionality of the radiation field, which is not taken into account by the Cauchy formula.

In order to calculate the path length for an incident ${ }^{12} \mathrm{C}$ ion beam, the SVs of the Mushroom detector were replaced with a vacuum to remove any physical interactions as particles traversed the SV, whilst maintaining the angular dependency of the radiation field incident on the microdosimetric device. The length of the track within the SV was recorded and the mean path length $\left\langle l_{\text {Path }}\right\rangle$ was then calculated and compared to the mean chord length $\left(\left\langle l_{\text {Cauchy }}\right\rangle\right)$ given by the Cauchy's formula which is $6.7 \mu \mathrm{m}$ for each SV of the Mushroom microdosimeter.

The kinetic energy of electrons entering the SVs was recorded and their CSDA range was determined in silicon using the NIST ESTAR database [13]. If the distance the electron travelled inside the detector was greater than the CSDA range then this event was classified as a stopper and not included in the determination of the chord length distribution and of $\left\langle l_{\text {Path }}\right\rangle$. The same method was used for ions however ions where found to have less impact on the mean path length than electrons in terms of being stoppers.

\section{4. $R B E_{10}$ Calculation}

The effect of adopting the investigated tissue-equivalence correction method and $\left\langle l_{\text {Path }}\right\rangle$ instead of $\left\langle l_{\text {Cauchie }}\right\rangle$ was benchmarked by calculating the $\mathrm{RBE}_{10}$ based on the Geant 4 simulated microdosimeter response against a spherical muscle SV with diameter of $10 \mu \mathrm{m}$, which was then compared to the $\mathrm{RBE}_{10}$ determined experimentally with TEPC measurements at HIMAC, NIRS [4]. 
A Modified MKM [4] was adopted to calculate the $\mathrm{RBE}_{10}$. In this approach the $\alpha$ parameter in the Linear Quadratic Model [15], which predicts the cell survival in a radiation field of interest, is a linear function of the saturation-corrected dose mean lineal energy, $y^{*}$, (equation (2)). The saturation parameter, $y_{0}$, is used to fit cell survival curves. Kase et al. [4] found that a $y_{0}$ equal to $150 \mathrm{keV} / \mu \mathrm{m}$ fitted survival data the best. The measurement of microdosimetric spectra at any depth in the phantom allows to derive $f(y)$ and derive $\mathrm{RBE}_{10}$ following equations (2) and (4). In this case human salivary glands were used with parameters being $r_{d}=0.42 \mu m$ (the radius of the domain), $\rho=1 \mathrm{~g} / \mathrm{cm}^{3}$ (density of cell's domain), $\alpha_{0}=0.13 G y^{-1}$ and $\beta=0.05 G y^{-2}$. Then the $\mathrm{RBE}_{10}$ using equation (4), where $D_{10, x-\text { ray }}$ is the dose required for $10 \%$ survival for $200 \mathrm{keV}$ $x$-rays and has a value of $5 \mathrm{~Gy}$.

$$
\begin{gathered}
y^{*}=y_{0}^{2} \frac{\int\left(1-\exp \left(-y^{2} / y_{0}^{2}\right)\right) f(y) d y}{\int y f(y) d y} \\
\alpha=\alpha_{0}+y^{*} \frac{\beta}{\rho \pi r_{d}^{2}} \\
R B E_{10}=\frac{2 \beta D_{10, x-r a y}}{\sqrt{\alpha^{2}-4 \beta \ln (0.1)}-\alpha}
\end{gathered}
$$

All vertical error bars shown in this work represent one standard deviation which come from the statistical fluctuation of ten different runs of the simulation. Horizontal error bars represent the bin width of the binned data.

\section{Results and Discussion}

\subsection{Tissue Equivalence}

Figure 2 shows the energy deposition spectra for silicon and muscle, for the depth of $130 \mathrm{~mm}$. Table 1 summarises the p-values, resulting from the comparison of the energy deposition spectra calculated in the $\mathrm{SV}_{S i}\left(l_{S i}=10 \mu \mathrm{m}\right)$ and $\mathrm{SV}_{\text {water/muscle }}$ with varying sizes of $l$.
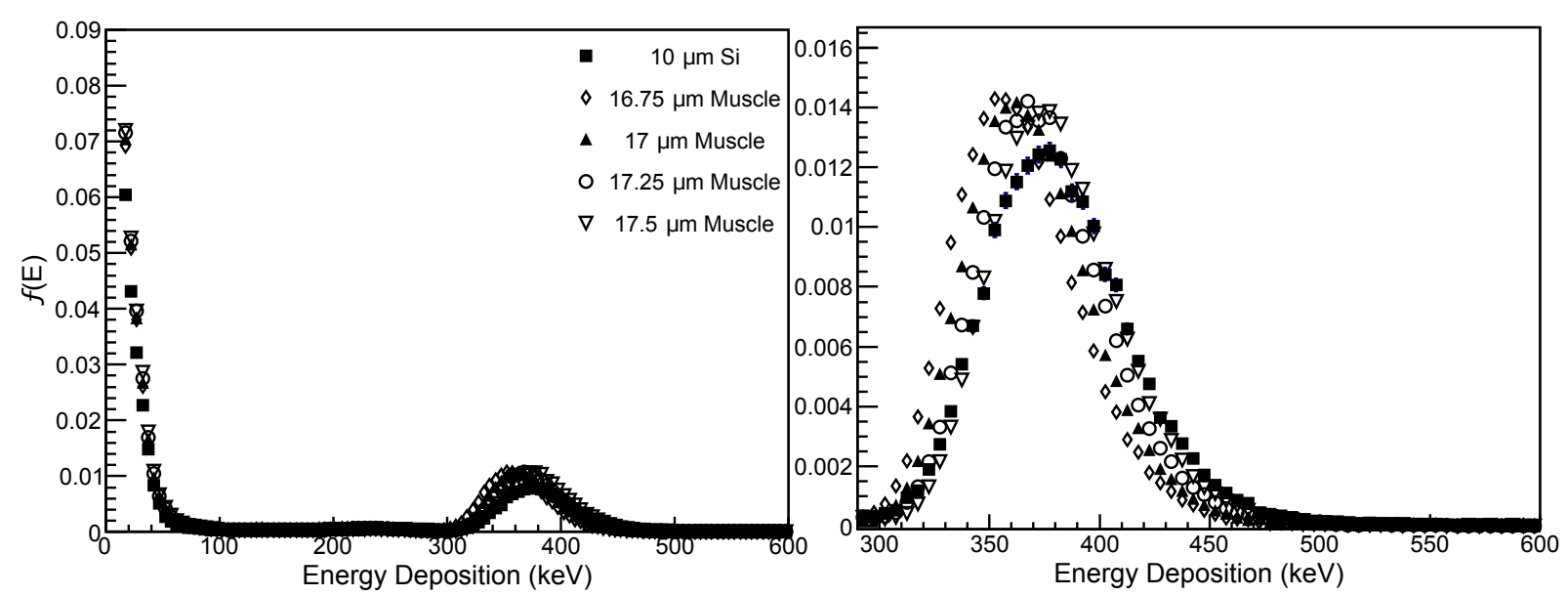

Figure 2: Energy deposition spectra obtained in $\mathrm{SV}_{S i}$ and in $\mathrm{SV}_{\text {muscle }}$ with varying size $l$ (indicated in the legend), at a depth of $130 \mathrm{~mm}$. The left represents the entire energy deposition distribution and the right plot shows a zoom in of the primary ${ }^{12} \mathrm{C}$ energy deposition peak.

As can be seen in Table 1, at 50 and $130 \mathrm{~mm}$ depth in the phantom the best agreement is obtained with $l_{\text {muscle }}$ equal to $17.5 \mu \mathrm{m}$, however at $160 \mathrm{~mm}$ all $l_{\text {muscle }}$ between 17 and $17.5 \mu \mathrm{m}$ do not differ significantly with the response in the silicon SV. For this reason a scaling factor $\kappa$ for 
muscle was chosen based on the SV $l_{\text {muscle }}$ equal to $17.5 \mu \mathrm{m}$, corresponding to a $\kappa$ of 0.57 . For water the best agreement for energy deposition spectra for silicon was found to be for $18.5 \mu \mathrm{m}$ corresponding to a $\kappa$ of 0.54 .

\begin{tabular}{c|cc|cc}
\hline Depth $(\mathrm{mm})$ & Muscle SV $l(\mu \mathrm{m})$ & p-value & Water SV $l(\mu \mathrm{m})$ & p-value \\
\hline 50 & 17 & $1.98 \mathrm{E}-08$ & 18 & 0.0405789 \\
$232 \mathrm{MeV} / \mathrm{u}$ & 17.25 & 0.775748 & 18.5 & 1 \\
& 17.5 & 1 & 19 & 0.239555 \\
\hline 130 & 17 & $1.85 \mathrm{E}-07$ & 18 & 0.156499 \\
$111 \mathrm{MeV} / \mathrm{u}$ & 17.25 & 0.990125 & 18.5 & 1 \\
& 17.5 & 1 & 19 & $2.46 \mathrm{E}-06$ \\
\hline 160 & 17 & $3.28 \mathrm{E}-03$ & 18 & 0.0774045 \\
$9 \mathrm{MeV} / \mathrm{u}$ & 17.25 & 0.995066 & 18.5 & 0.85756 \\
& 17.5 & 0.986345 & 19 & $1.66 \mathrm{E}-18$ \\
\hline
\end{tabular}

Table 1: p-values calculated using the $\chi^{2}$ test comparing the energy deposition distribution of $\mathrm{SV}_{S i}$ to $\mathrm{SV}_{\text {muscle/water }}$ with different sizes $l$.

Figure 3 shows the energy deposition spectra up to $50 \mathrm{keV}$ in muscle and silicon SVs at $50 \mathrm{~mm}$ depth in the phantom, which is due to electrons originating from outside of the SV, reaching the SV and depositing energy as well as electrons being generated by photons inside the detector. It can be observed that silicon shows a higher frequency of low energy deposition events (up to few $\mathrm{keV}$ ) with respect to muscle (as well as water), which means that a higher number of electrons deposit energy in the $\mathrm{SV}_{S i}$. The extra electrons in the $\mathrm{SV}$ derive from photon interactions as the attenuation coefficient in silicon is about 10 times higher than in water/muscle for energies $>100 \mathrm{keV}$, as shown in Figure 3 .
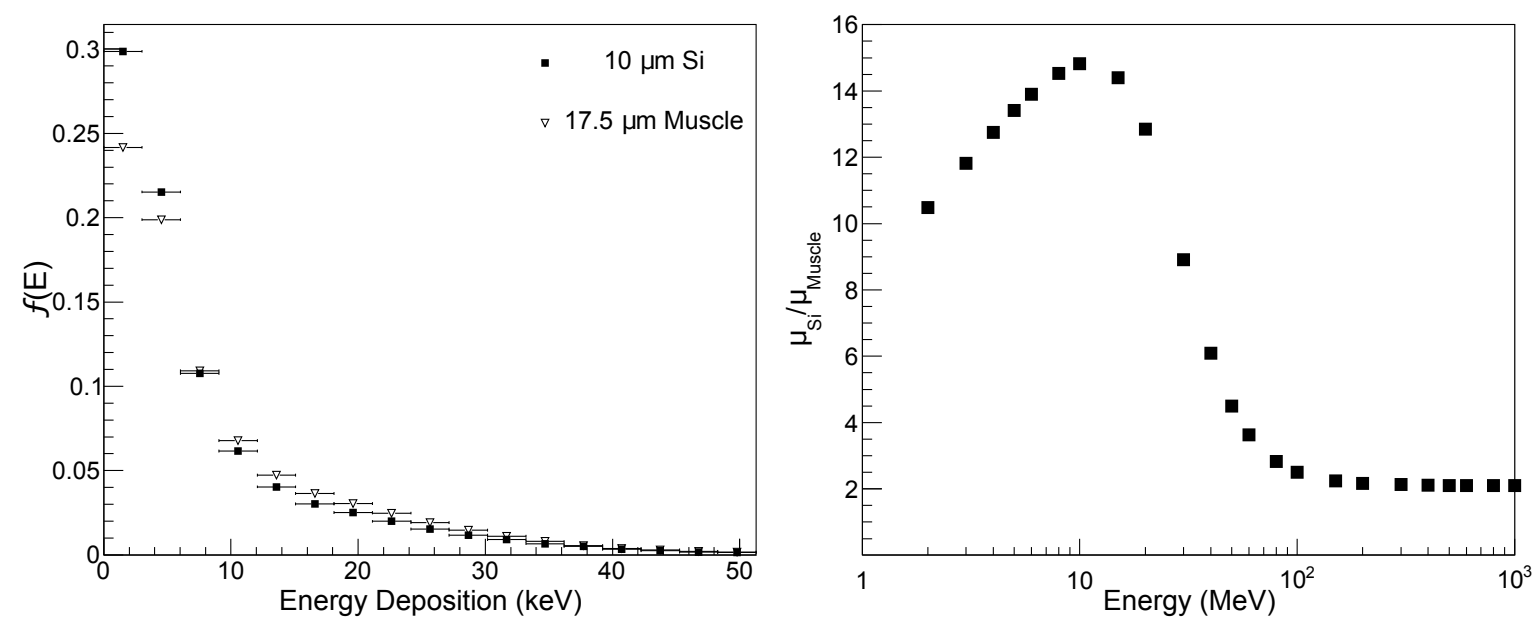

Figure 3: Left: A zoomed in figure showing the over response of silicon at low energy deposition due to different stopping powers of electrons, the legend indicates the size and material of the SV. Right: plot of the ratio of the linear attenuation coefficients in silicon and muscle [16].

A $L E C$ (Low Energy Correction) factor was introduced to take into account the over response of silicon and to make the $\mathrm{SV}_{S i}$ response more similar to water/muscle. The energy deposition 
spectrum in the $\mathrm{SV}_{S i}$ between zero and an energy threshold $\mathrm{E}_{t}$ is scaled by the $L E C$ factor as shown in Equation 5. Equation (5) shows the number of counts of a corrected silicon spectrum for all energies $\left(N\left(E_{\text {SiCorr }}\right)\right)$ being the addition of the counts $\left(N_{1}\right)$ in the energy range of 0 to the threshold energy $\left(N_{1}\left(E_{S i}\right)\right)$, scaled by the $L E C$ factor, plus the counts $\left(\mathrm{N}_{2}\right)$ above the threshold energy to the maximum energy of the spectrum $\left(\mathrm{E}_{\max }\right)$.

$$
N\left(E_{\text {SiCorr }}\right)=(L E C)\left(N_{1}\left(\left.E_{S i}\right|_{0} ^{E_{t}}\right)\right)+\left.N_{2}\right|_{E_{t}} ^{E_{\max }}
$$

The $L E C$ and $\mathrm{E}_{t}$ were determined ad hoc to provide best agreement between the $y_{F}$ calculated in muscle and silicon SVs. Table 2 shows the $L E C$ factor and the $\mathrm{E}_{t}$ with respect to the depth in the phantom and the corresponding mean kinetic energy of the ${ }^{12} \mathrm{C}$ incident beam at the specific depth. The $L E C$ factors were found to be the same for muscle and water SVs since the ratio of attenuation coefficients of water and muscle is $\sim 1$.

In order to apply these corrections for other setups they may be applied based upon the mean energy of the carbon peak of the deposited energy spectrum (MCA) as shown in table 2.

\begin{tabular}{cccc}
\hline Depth in Phantom $(\mathbf{m m})$ & Mean of Carbon peak $(\mathrm{keV})$ & $E_{t}(\mathrm{keV})$ & LEC \\
\hline$<50$ & $<237$ & 15 & 0.8 \\
$50-140$ & $\geq 237$ & 20 & 0.73 \\
$>140$ & $>481$ & 30 & 0.73 \\
$>160$ & $n / a$ & 15 & 0.8 \\
\hline
\end{tabular}

Table 2: $L E C$ factors to be applied up to the energy threshold $E_{t}$, based on the depth in the phantom, corresponding to the energy deposition peak produced by the carbon ion beam. Note the $n / a$ corresponds to downstream of the BP.

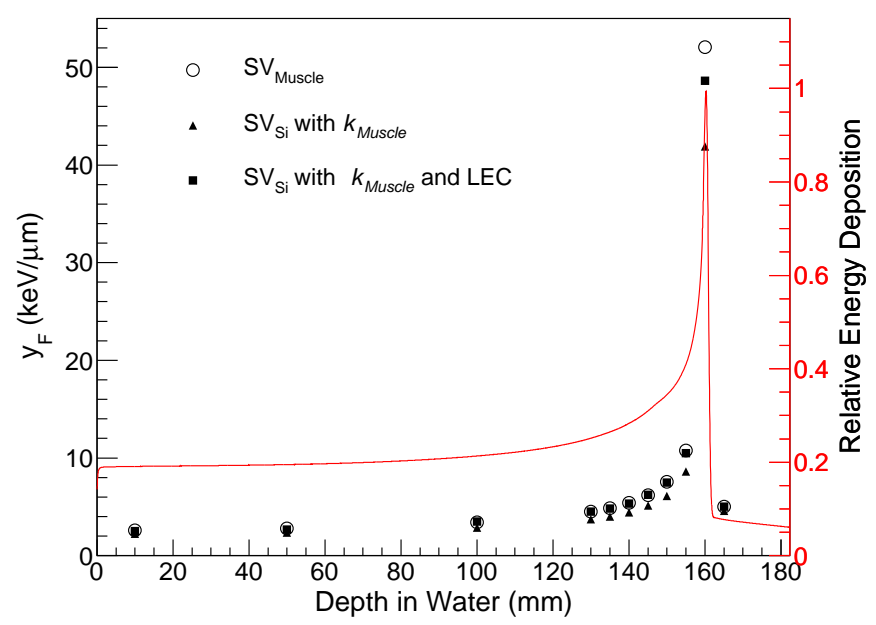

Figure 4: Plot showing $y_{F}$, calculated with a muscle SV (open circles), a silicon SV with $\kappa_{m u s c l e}$ correction only (triangle), and with both $\kappa_{\text {muscle }}$ and LEC corrections(square).

Figure 4 summarises the effect of the $L E C$ on the microdosimetric quantity $y_{F}$, which is seen to improve $\sim 10 \%$ by reducing the contribution of low energy deposition events in the $\mathrm{SV}_{S i}$. The value $y_{D}$ was seen to improve only minutely due to it being weighted for higher energy events. 

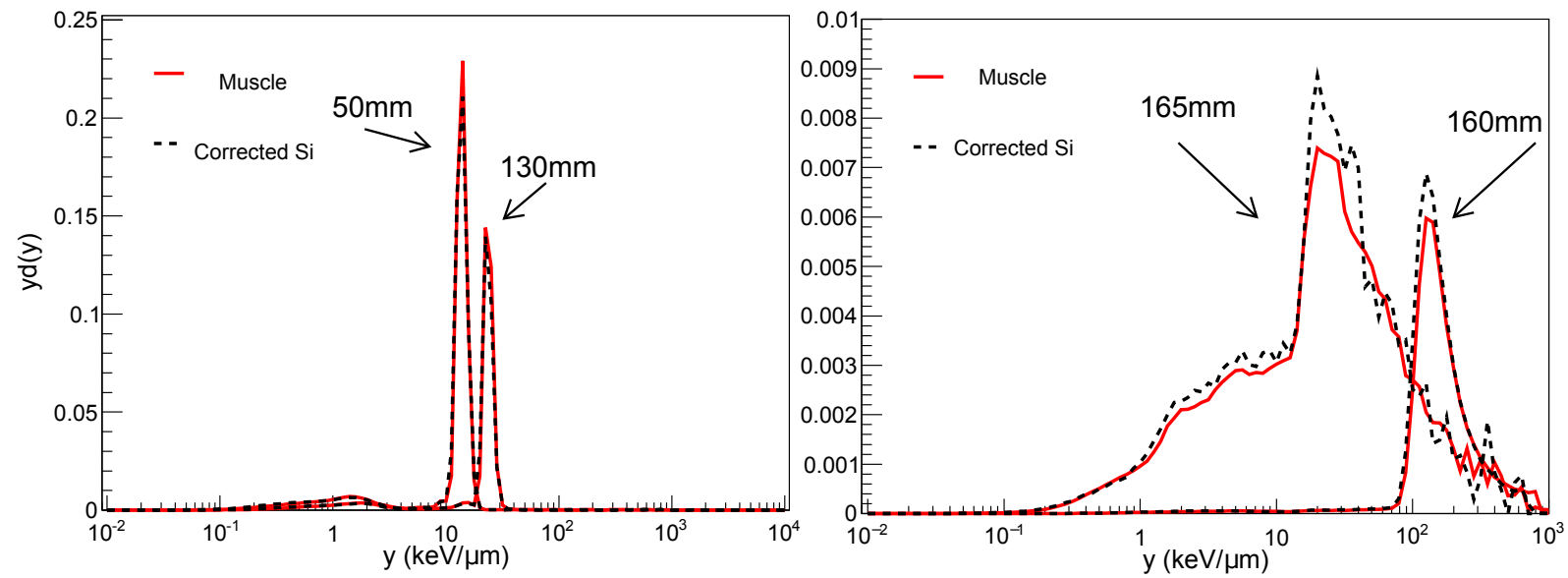

Figure 5: Comparison of the microdosimetric spectra calculated in muscle SVs and the corrected spectra in the silicon SVs, at depths in phantom equal to: 50, 130, 160 and $165 \mathrm{~mm}$.

Figure 5 shows the dose weighted distributions, corrected by $\kappa$ and LEC for muscle. It can be observed that there is an excellent alignment of peaks of the spectra, even after all the incident ${ }^{12} \mathrm{C}$ ions have stopped and only secondary fragments remain (corresponding to a depth of 165 $\mathrm{mm})$.

\subsection{Study on the path length distribution and mean path length}

Figure 6 shows how the path length distributions (PLD) vary at particular depths including: near the phantom entrance $(10 \mathrm{~mm})$, at the $\mathrm{BP}(160 \mathrm{~mm})$ and distally from the $\mathrm{BP}(165 \mathrm{~mm})$. The path length is presented for primary ${ }^{12} \mathrm{C}$ ions and for secondary particles originated outside of the SV and traversing the detector. The right figure shows the PLDs at the BP for different particle types. It can be seen that the primary and secondary ions both have peaks at $10 \mu \mathrm{m}$ corresponding to particles entering perpendicular with the face of the SV, while electrons do not have this peak as they have a more isotropic distribution.
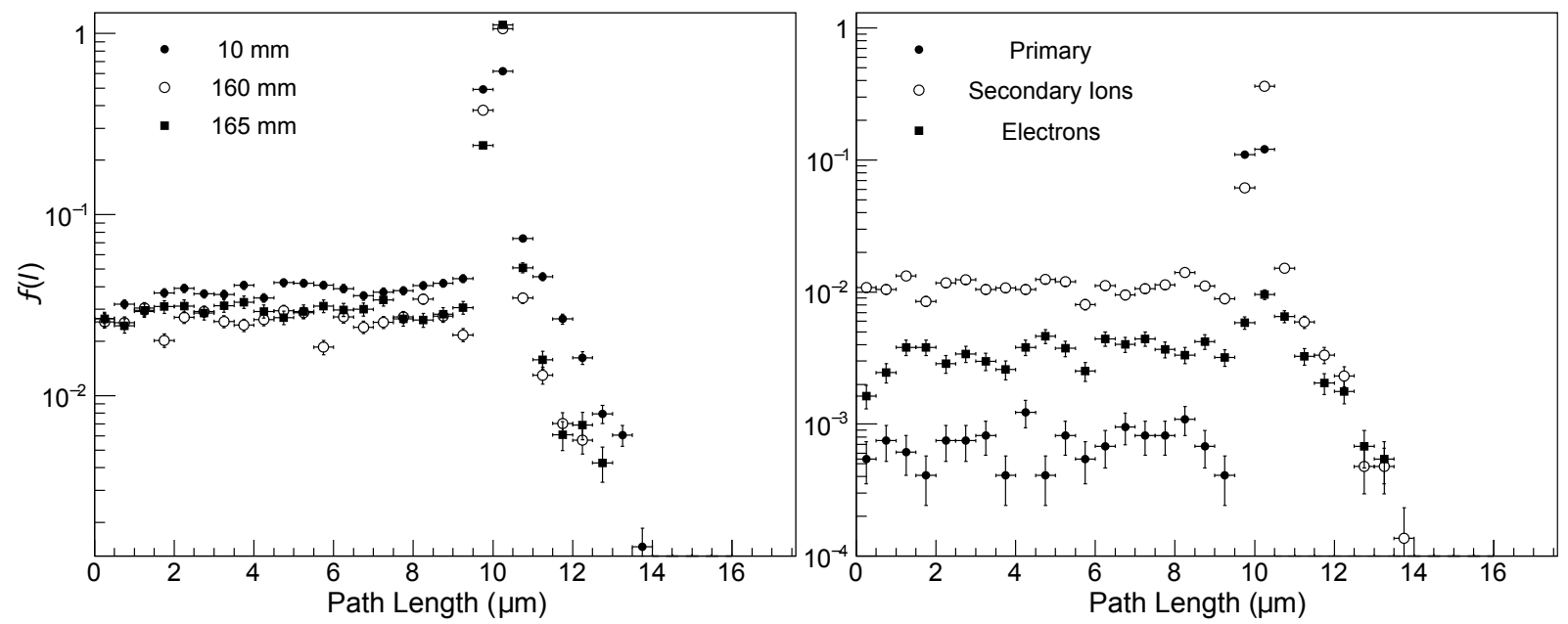

Figure 6: Left: PLDs for all particles at the entrance of the phantom $(10 \mathrm{~mm})$ at the BP (160 $\mathrm{mm})$ and distal to the BP $(165 \mathrm{~mm})$. Right: The path length distributions for different particles at the BP. 
Figure 7 shows the $\left\langle l_{\text {Path }}\right\rangle$ along the depth in the phantom. The mean path lengths are then shown for secondary ions and electrons, separately. The incident carbon ions have a strong directionality therefore their $\left\langle l_{\text {Path }}\right\rangle$ does not vary much and is approximately the same as the thickness of the SV $(10 \mu \mathrm{m})$ in the direction of the incident ${ }^{12} \mathrm{C}$ beam. The $\left\langle l_{\text {Path }}\right\rangle$ of electrons is approximately $6.7 \mu \mathrm{m}$ for all depths in the phantom and agrees well with $\left\langle l_{\text {Cauchy }}\right\rangle$, which is characteristic for an isotropic field. The $\left\langle l_{\text {Path }}\right\rangle$ of secondary ions varies largely in comparison to other types of particles such as the incident carbon ions and secondary electrons. At shallow depths in the phantom, $\left\langle l_{\text {Path }}\right\rangle$ is approximately $8.5 \mu \mathrm{m}$ and then increases continuously up to the BP. This happens because of neutrons, which scatter significantly in water and are produced mainly at the surface of the phantom. Neutrons scatter target nuclei of the medium being mostly $\mathrm{H}$ for water, which then have a comparatively large angular distribution, thus producing a $\left\langle l_{\text {Path }}\right\rangle$ closer to the one produced by an isotropic field. On the other hand, fragments produced from the interaction of the primary ${ }^{12} \mathrm{C}$ with target nuclei have a tendency to be more forwarded directed. This means that at increasing depths in the phantom where the neutron production decreases and the fragment production stays more consistent which causes the $\left\langle l_{\text {Path }}\right\rangle$ to steadily increase with depth in the phantom.

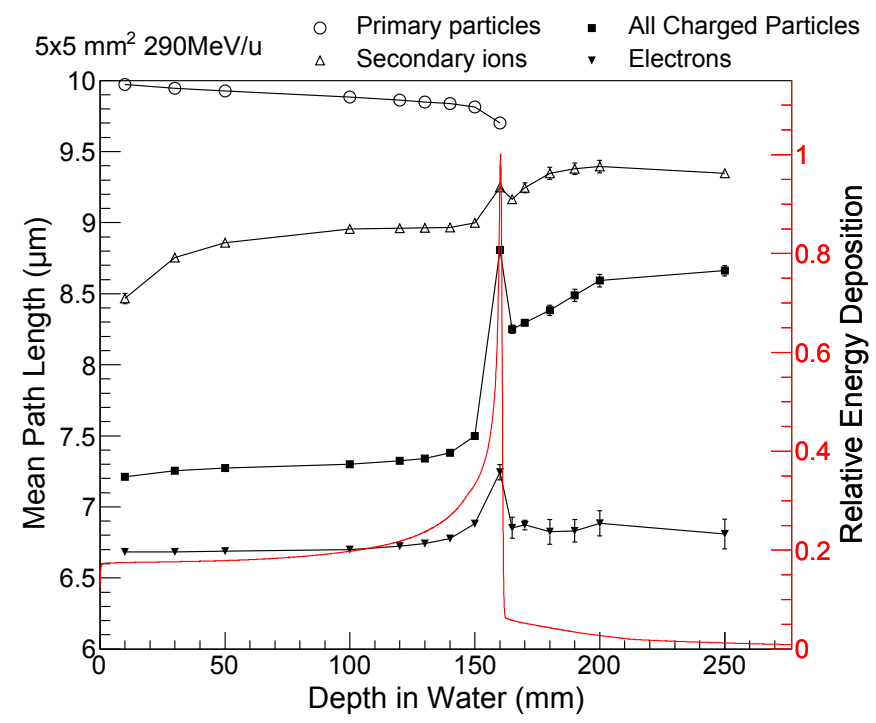

Figure 7: $\left\langle l_{\text {Path }}\right\rangle$ of different particles traversing the $10 \mu \mathrm{m}$ cylinder volume array at various depths in phantom. The SV is filled as a vacuum. The Bragg Curve is shown as well for reference.

Beyond the BP there is a small decrease in $\left\langle l_{\text {Path }}\right\rangle$ for secondary ions, which is due to a sharp decrease in fragmentation production due to the incident carbon ion beam having been stopped, increasing neutron contribution. Further downstream of the BP $\left\langle l_{\text {Path }}\right\rangle$ can be seen to increase, this is due to more laterally directed particles leaving the centre of the beam, at greater depths. This causes the more forward directed particles to be more dominant.

The $\left\langle l_{\text {Path }}\right\rangle$ for secondary ions was studied with respect to the ${ }^{12} \mathrm{C}$ ion beam sizes of $5 \times 5 \mathrm{~mm}^{2}$ and $50 \times 50 \mathrm{~mm}^{2}$, the results are shown in figure 8 . It can be observed that the $\left\langle l_{\text {Path }}\right\rangle$ of lighter secondary nuclear fragments decreases with larger field sizes. This happens because in larger beam sizes lighter fragments such as $\mathrm{H}$ have a higher probability to be scattered into the centre of the beam where the detector is positioned, contributing to a more isotropic field. Thus the mean path length value becomes closer to $\left\langle l_{\text {Cauchy }}\right\rangle$.

Figure 9 (a) depicts the $\left\langle l_{\text {Path }}\right\rangle$ against the average kinetic energy of the primary beam when reaching the SV for each different primary beam energies of $150 \mathrm{MeV} / \mathrm{u}, 290 \mathrm{MeV} / \mathrm{u}$ and 400 

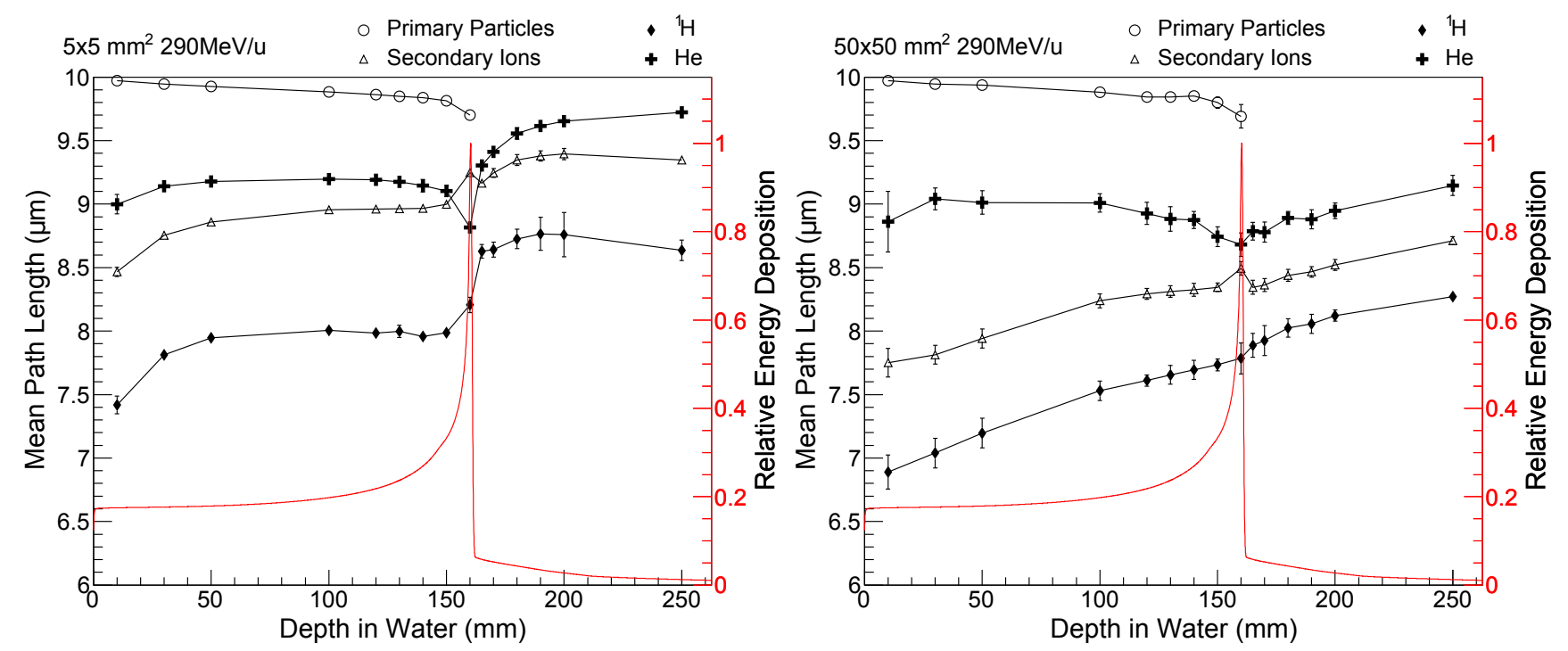

Figure 8: Comparison of mean path lengths for different particles for $5 \times 5 \mathrm{~mm}^{2}$ and $50 \times 50$ $\mathrm{mm}^{2}$ fields sizes. The incident ${ }^{12} \mathrm{C}$ energy was $290 \mathrm{MeV} / \mathrm{u}$.

$\mathrm{MeV} / \mathrm{u}$. The $\left\langle l_{\text {Path }}\right\rangle$ of secondary electrons, which is not plotted, is approximately constant and equal to $6.7 \mu \mathrm{m}$. Figure 9 (b) illustrates the concept of calculating $\left\langle l_{\text {Path }}\right\rangle$ using the energy of the ${ }^{12} \mathrm{C}$ beam in the phantom instead of the depth.

The $\left\langle l_{P a t h}\right\rangle$ of the primary beam for different initial beam energies are very similar for the same energy of the primary carbon beam. For secondary ions, at depths close to the surface of the phantom, in the case of the higher energies of the incident beam, the $\left\langle l_{\text {Path }}\right\rangle$ for secondary ions changes rapidly due to the changing contribution of recoiled nuclei and fragment products. It is seen that beyond an average primary beam energy of $234 \mathrm{MeV} / \mathrm{u}$ that $290 \mathrm{MeV} / \mathrm{u}$ and $400 \mathrm{MeV} / \mathrm{u}$ values are within error. The incident $150 \mathrm{MeV} / \mathrm{u}^{12} \mathrm{C}$ ion beam produces fewer fragments relative to neutrons compared to $290 \mathrm{MeV} / \mathrm{u}$ and $400 \mathrm{MeV} / \mathrm{u}$, leading to a smaller $\left\langle l_{\text {Path }}\right\rangle$. This leads to the $\left\langle l_{\text {Path }}\right\rangle$ being approximately $0.5 \mu \mathrm{m}$ smaller than in the case of $290 \mathrm{MeV} / \mathrm{u}$ and $400 \mathrm{MeV} / \mathrm{u}$ beams at the same average primary beam energy when reaching the SV.

These results are useful for this specific experimental context. As without knowing the initial energy of the ${ }^{12} \mathrm{C}$ ion beam, the mean path length at any arbitrary depth in a phantom can be estimated fairly accurately for the primary beam using only the energy deposition spectra in the microdosimeter (like shown in figure 2). Additionally, provided that the beam energy is high enough $(\sim>150 \mathrm{MeV} / \mathrm{u})$, the $\left\langle l_{\text {Path }}\right\rangle$ of the secondary ions can be determined. This is found to be valid starting from a depth in the phantom of a few $\mathrm{cm}$, when the kinetic energy of the primary beam has decreased by about $30 \mathrm{MeV} / \mathrm{u}$ (approximately $30 \mathrm{~mm}$ for $290 \mathrm{MeV} / \mathrm{u}$ ). Such a method has the advantage to be easily applicable in experimental practice as the energy deposition spectra are directly measured by the microdosimeter and can be analysed.

Table 3 summarises the $\left\langle l_{\text {Path }}\right\rangle$ for this particular HIT radiation field studied, including incident carbon ions and nuclear recoils, based upon the value of the peak produced by incident ${ }^{12} \mathrm{C}$ ions in the energy deposition spectrum. The mean path length for secondary ions assumes that the primary beam energy is $>150 \mathrm{MeV} / \mathrm{u}$ in order to produce a similar amount of fragments as $290 \mathrm{MeV} / \mathrm{u}$ and $400 \mathrm{MeV} / \mathrm{u}$.

For the distal part of the BP, Table 3 cannot be used to determine the mean path length as all the incident ${ }^{12} \mathrm{C}$ ions have been stopped. However, the mean path length of the secondary radiation field is very similar downstream of the BP for each primary energy and after approximately $20 \mathrm{~mm}$ downstream $\left\langle l_{\text {Path }}\right\rangle$ remains mostly unchanged for all energies with 
(a)

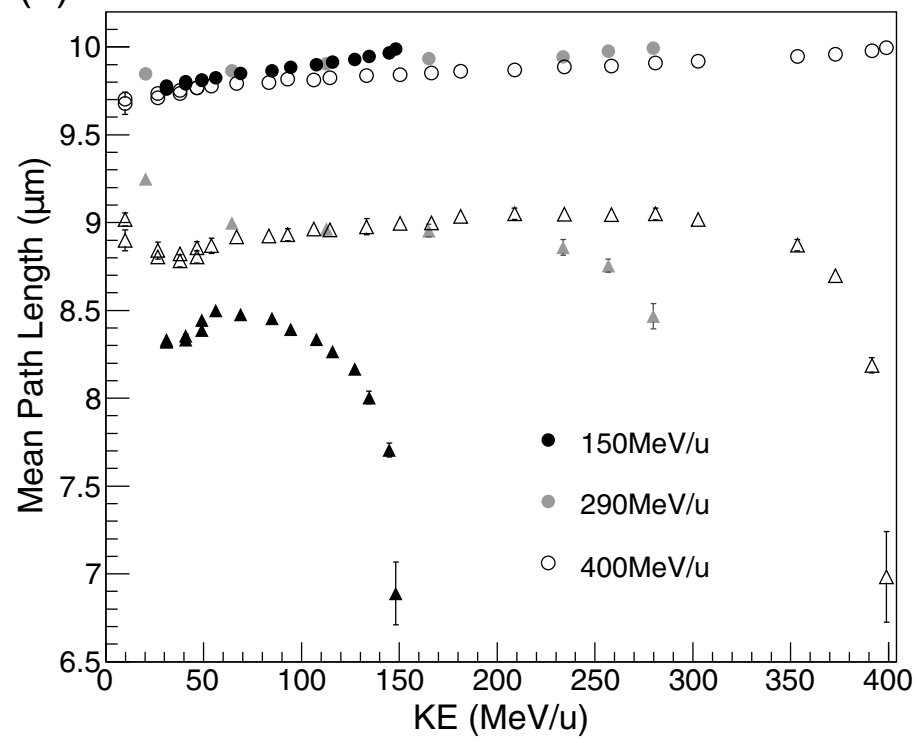

(b)

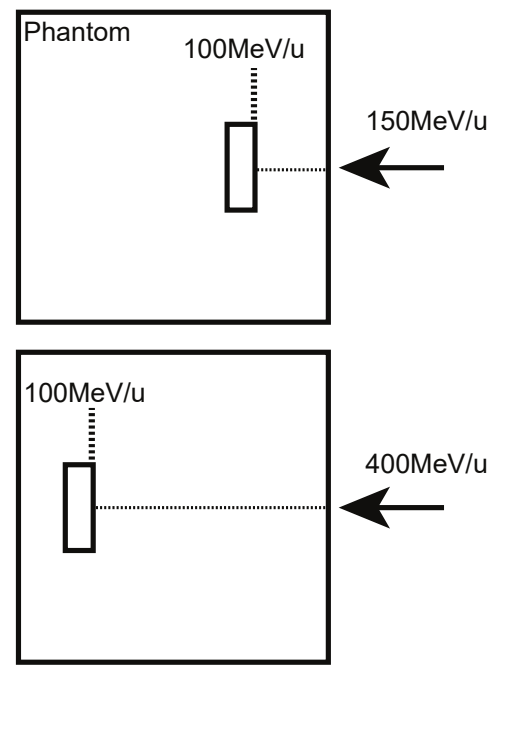

Figure 9: (a) Comparison of the mean path length based on the average kinetic energy of the ${ }^{12} \mathrm{C}$ ion beam at specific depths in the phantom, for different incident energies as indicated in the legend. The circle markers represent the $\left\langle l_{\text {Path }}\right\rangle$ of the primary ${ }^{12} \mathrm{C}$ beam while the triangle markers represent $\left\langle l_{\text {Path }}\right\rangle$ of secondary ions. (b) Diagram demonstrating how the $\left\langle l_{\text {Path }}\right\rangle$ is derived based on the energy of the primary beam at specific depths.

\begin{tabular}{c|c|c}
\hline $\begin{array}{c}\text { Mean }{ }^{12} \mathbf{C} \text { energy } \\
\text { deposition peak (keV) }\end{array}$ & Primary $\left\langle l_{\text {Path }}\right\rangle(\mu \mathbf{m})$ & Secondary Ions $\left\langle l_{\text {Path }}\right\rangle(\mu \mathbf{m})$ \\
\hline 180 & 10.0 & 8.2 \\
183 & 10.0 & 8.7 \\
188 & 9.9 & 8.9 \\
207 & 9.9 & 9.0 \\
327 & 9.8 & 9.0 \\
472 & 9.8 & 8.9 \\
822 & 9.8 & 8.8 \\
988 & 9.7 & 8.8 \\
2973 & 9.7 & 9.0 \\
\hline
\end{tabular}

Table 3: Mean path length based on the peak produced by the incident $5 \times 5 \mathrm{~mm}^{2}$ carbon ions in the energy deposition spectrum (called here the mean ${ }^{12} \mathrm{C}$ energy deposition Peak). The secondary ions $\left\langle l_{\text {Path }}\right\rangle$ are based on $290 \mathrm{MeV} / \mathrm{u}$ and $400 \mathrm{MeV} / \mathrm{u}$. Electrons (not included here) have a $\left\langle l_{\text {Path }}\right\rangle$ of $6.7 \mu \mathrm{m}$ essentially for all energies.

a value of $\sim 9.4 \mu \mathrm{m}$.

These results are for this particular radiation field and microdosimeter, they show how the $\left\langle l_{\text {Path }}\right\rangle$ varies when changing certain quantities of field size and energy. For accurate results $\left\langle l_{\text {Path }}\right\rangle$ calculations should be performed using the particular radiation field and microdosimeter of interest. Additionally, these results simulate the case of perfect alignment of the microdosimeter within the phantom, since the current practical method of positioning the 
microdosimeter in the phantom has been seen to be robust. However future work will be to determine the dependency of the $\left\langle l_{\text {Path }}\right\rangle$ due to possible misalignment of the microdosimeter in the phantom; as well as explore the more clinical case of a SOBP radiation field.

Summarising, the results of this research show that the mean path length varies quite noticeably for different components of the HIT radiation field, most pronounced between the primary carbon beam and electrons by $\sim 30 \%$ for different depths in the phantom. Recoiled protons have a wider angular spread, while incident carbon ions and fragmented ions go largely straight through the detector.

To obtain a more constant mean path length for components of the radiation field, the SV should have dimensions with the thickness of the SV along the beam direction equal to $\left\langle l_{\text {Cauchy }}\right\rangle$. So in the case of a cylindrical SV like the Mushroom the height should be half the diameter of the SV. The resulting $\left\langle l_{\text {Path }}\right\rangle$ are shown in figure 10 for a $20 \times 20 \mathrm{~mm}^{2}$ field which is between the two extreme cases of $5 \times 5 \mathrm{~mm}^{2}$ and $50 \times 50 \mathrm{~mm}^{2}$. It can be seen that the mean path length values are between $9 \mu \mathrm{m}$ and $10 \mu \mathrm{m}$ for all components of the radiation field. This design is recommended for hadrontherapy where the radiation field is directional.

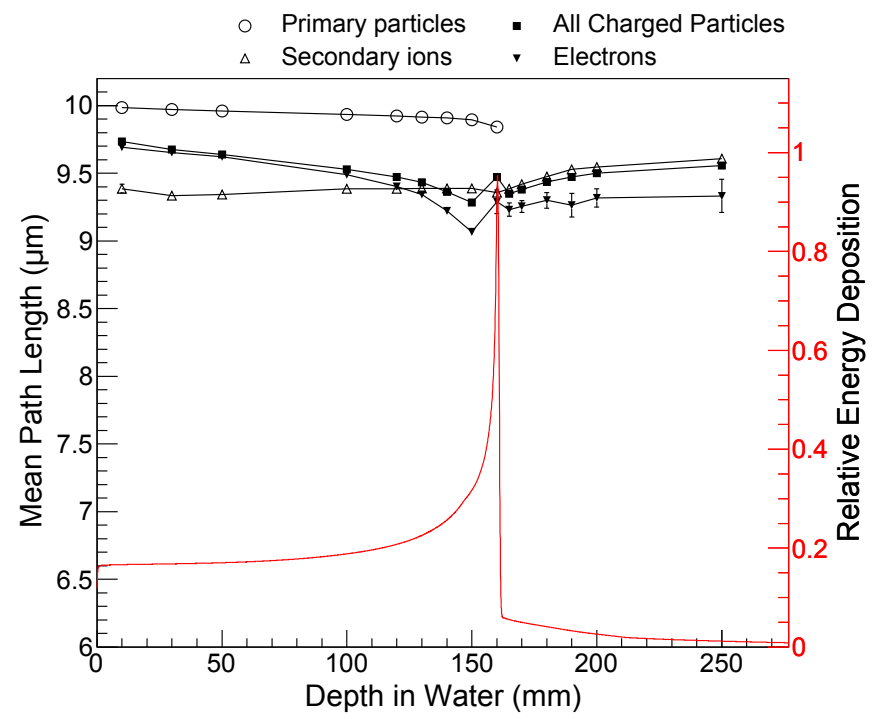

Figure 10: Calculated mean path length of particles traversing the cylinder of height $10 \mu \mathrm{m}$ and diameter $20 \mu \mathrm{m}$, using a $20 \times 20 \mathrm{~mm}^{2}$ primary beam with energy of $290 \mathrm{MeV} / \mathrm{u}$.

\section{3. $R B E_{10}$ Determination}

Figure 11 shows the $\mathrm{RBE}_{10}$ calculated by means of the Geant 4 simulations in the Mushroom device with silicon SVs. The correction factors $\kappa$ and $L E C$ have been adopted to convert the microdosimetric spectra in silicon to muscle. The $\mathrm{RBE}_{10}$ calculated in the Mushroom with SVs made of muscle are also shown in figure 11, together with the $\mathrm{RBE}_{10}$, determined from experimental measurements performed at HIMAC [4], with a $12.5 \mathrm{~mm}$ diameter TEPC.

It can be observed that the $\mathrm{RBE}_{10}$ calculated in silicon and muscle SVs agree thanks to the adopted corrections. A good agreement is also observed with the experimental $\mathrm{RBE}_{10}$ determined with a TEPC, slight discrepancies can be attributed in part due to the differences of the beam lines. This means that in principle the Mushroom detector can be adopted to determine the $\mathrm{RBE}_{10}$, but with an additional high spatial resolution, which cannot be achieved with the TEPC. 

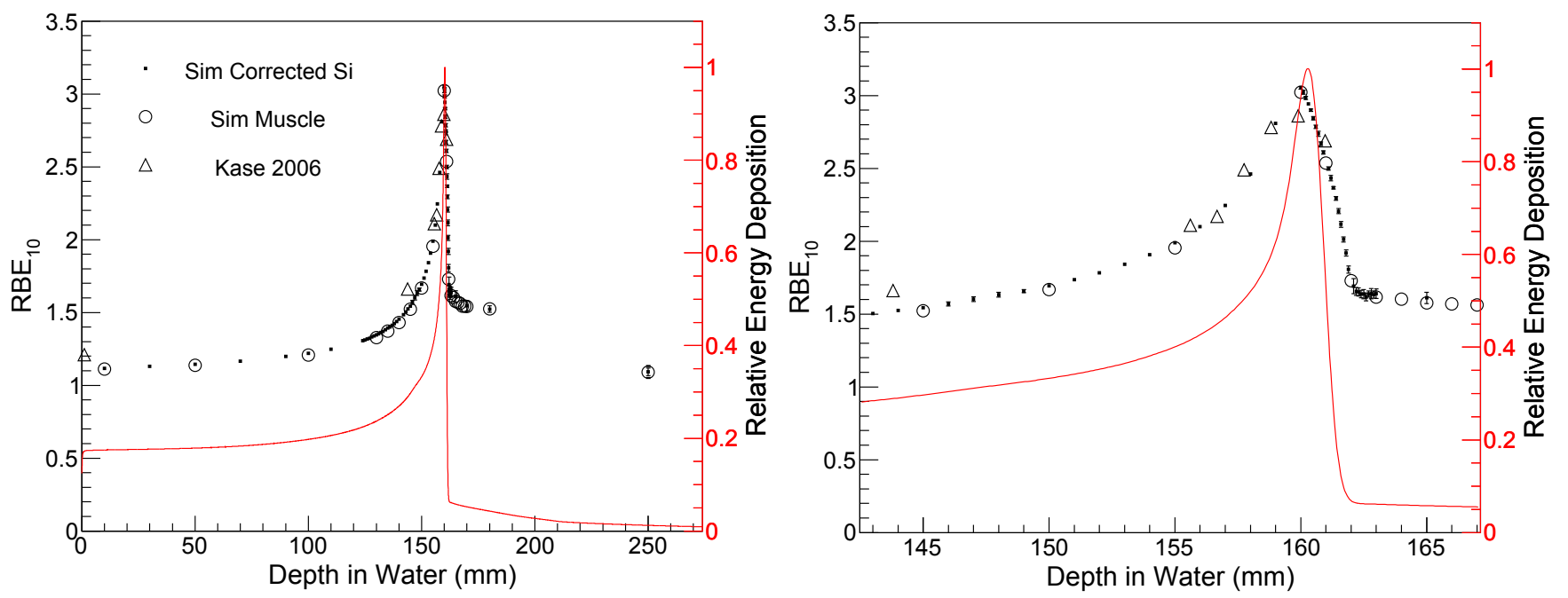

Figure 11: Left: Calculated $\mathrm{RBE}_{10}$ along the depth in water, using the Mushroom detector. The $\mathrm{RBE}_{10}$ resulting from the simulation study is compared to experimental measurements performed with the TEPC documented in Kase et al. [4]. Right: zoomed view of the results at the BP position.

To determine the importance of the $\langle l\rangle$ on calculated $\mathrm{RBE}_{10}$ values, three different $\mathrm{RBE}_{10}$ calculations were performed, with the results shown in figure 12. The different methods included applying the isotropic value of $6.7 \mu \mathrm{m}$ provided by Cauchy's formula as well as using the thickness of the SV, $10 \mu \mathrm{m}$. The remaining method was to use the values of $\left\langle l_{\text {Path }}\right\rangle$ found in table 3 of section 3.2, considering the specific chord length for each component of the radiation field (incident carbon ions, secondary ions and electrons). These different methods were compared to the simulated $\mathrm{RBE}_{10}$ of a simulated muscle sphere with a diameter of $10 \mu \mathrm{m}$ and $\left\langle l_{\text {Cauchy }}\right\rangle$ of $6.7 \mu \mathrm{m}$.

It can be seen in figure 12 that using the $\left\langle l_{\text {Cauchy }}\right\rangle$ of $6.7 \mu \mathrm{m}$ gives significantly different results compared to the other methods and notably differs from the sphere values and should not be used. The remaining two methods result in very similar $\mathrm{RBE}_{10}$ values. Such agreement is due to the fact that the primary ${ }^{12} \mathrm{C}$ dominates the radiation field with its high LET while the secondary particles contribute less significantly to the $\mathrm{RBE}_{10}$. The $\mathrm{RBE}_{10}$ determined with the Si SV is similar to the one calculated with the muscle sphere of $10 \mu \mathrm{m}$ diameter, with a difference of $\sim 2 \%$ which should be due to the different shape of the Si SV and muscle sphere. Larger differences are observed at the BP due to the sensitivity of $\mathrm{y} *$. Beyond the BP the greatest difference is found due to the the primary carbon beam being stopped. Because the ${ }^{12} \mathrm{C}\left\langle l_{\text {Path }}\right\rangle$ is very close to $10 \mu \mathrm{m}$ for all depths, very little difference is seen between using the calculated $\left\langle l_{\text {Path }}\right\rangle$ values and the thickness of the SV. Beyond the BP, the secondary ion's $\left\langle l_{\text {Path }}\right\rangle$ approaches $9.4 \mu \mathrm{m}$ (section 3.2) which is close to the thickness of the SV along the direction of the beam. This means that a constant $10 \mu \mathrm{m}\left\langle l_{\text {Path }}\right\rangle$ can be adopted instead of $\left\langle l_{\text {Path }}\right\rangle$ with no significant differences in calculated $\mathrm{RBE}_{10}$.

Because of the dominance of the primary ${ }^{12} \mathrm{C}$ and that the secondary ions have a $\left\langle l_{\text {Path }}\right\rangle$ value very close to the thickness of the SV beyond the BP makes it conveniently accurate to use the thickness of the SV as the $\left\langle l_{\text {Path }}\right\rangle$ value. However this applies to this setup of detector and beam using ${ }^{12} \mathrm{C}$ and may not be so accurate in proton therapy where protons can scatter more frequently, contributing to a more isotropic field and more importantly has much fewer fragments produced, causing downstream of the BP to be dominated by nuclei scattered by neutrons which should produce values close to $\left\langle l_{\text {Cauchy }}\right\rangle$. For simplicity, future solid state microdosimeter designs 
should adopt a thickness along the direction of the beam equal to the $\left\langle l_{\text {Cauchy }}\right\rangle$.
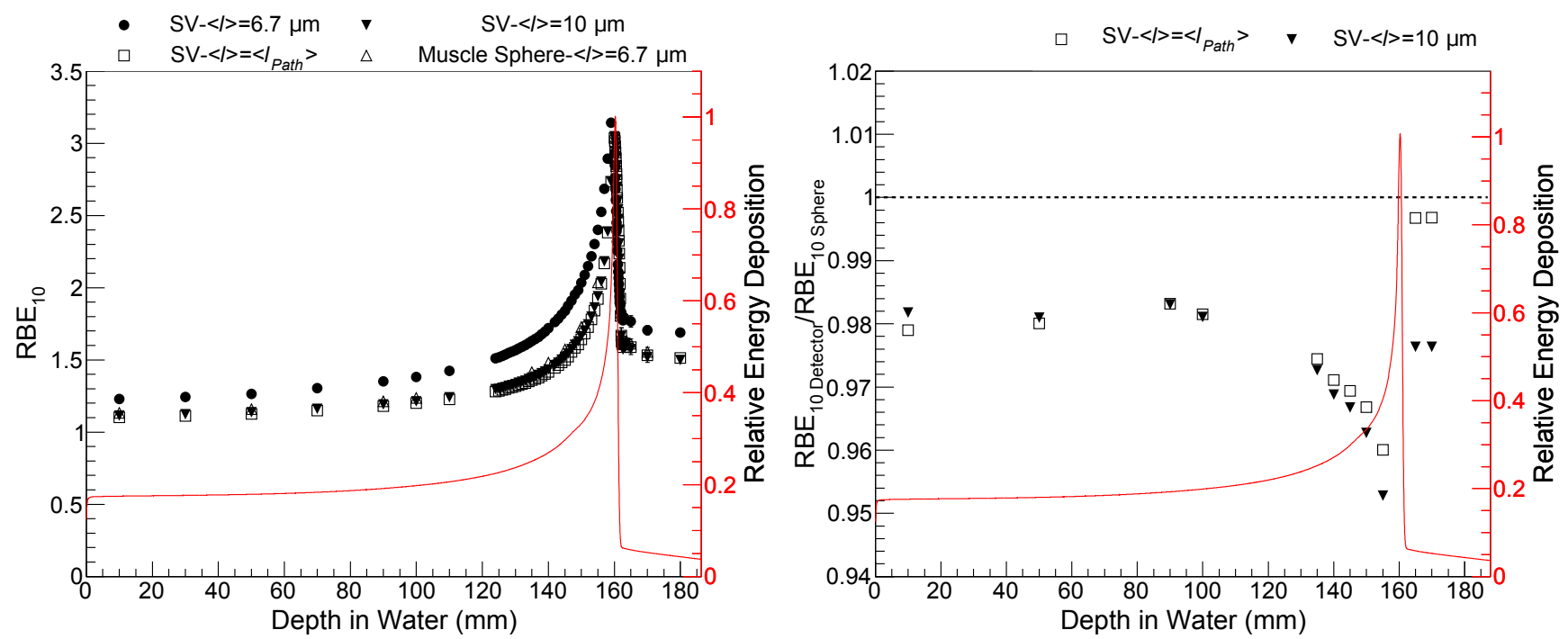

Figure 12: Left: $\mathrm{RBE}_{10}$ derived from the Si microdosimeter calculated using different $\langle l\rangle$ : the isotropic chord length of $6.7 \mu \mathrm{m}$, a constant $\langle l\rangle$ of $10 \mu \mathrm{m}$ representing the thickness of the SV and using the $\left\langle l_{\text {Path }}\right\rangle$ values found in section 3.2 for each particle type. These are compared against the $\mathrm{RBE}_{10}$ of a $10 \mu \mathrm{m}$ muscle sphere with a $\langle l\rangle$ of $6.7 \mu \mathrm{m}$. Right: The ratio of the $\mathrm{RBE}_{10}$ values of the microdosimeter using the $\langle l\rangle$ of $10 \mu \mathrm{m}$ and $\left\langle l_{\text {Path }}\right\rangle$ with the values of the muscle sphere.

\section{Conclusions}

An upcoming fully 3D CMRP silicon microdosimeter was studied using Geant4 in a pristine $290 \mathrm{MeV} / \mathrm{u}{ }^{12} \mathrm{C}$ ion beam. A correction factor of 0.57 was calculated to convert the response in silicon to that of muscle and similarly a value of 0.54 for water, with an additional low energy correction factor to address the over-response of silicon at low energies which varied with energy.

An alternative method to the mean chord length was used in the form of the mean path length. The adoption of $\left\langle l_{\text {Cauchy }}\right\rangle$ was demonstrated to be less accurate than the adoption of $\left\langle l_{\text {Path }}\right\rangle$ equal to the thickness of the SV, when determining the $\mathrm{RBE}_{10}$. This is due to the high directionality of the radiation field produced by the carbon ions.

It was demonstrated that the calculated correction factors allow to determine the $\mathrm{RBE}_{10}$ with good agreement with respect to the corresponding determined based on TEPC measurements.

Future designs of microdosimeters intended for use in hadron therapy should adopt a SV with thickness along the direction of the incident beam equal to $\left\langle l_{\text {Cauchy }}\right\rangle$.

\section{Acknowledgements}

We would like to thank the University of Wollongong Information Technology Services (ITS) for computing time on the UOW High Performance Computing Cluster. This project was partially funded by the Australian Research Council (ARC) Discovery Project grant number DP1096600.This research is supported by an Australian Government Research Training Program (RTP) Scholarship.

\section{References}

[1] M. Torikoshi, S. Minohara, N. Kanematsu, M. Komori, M. Kanazawa, K. Noda, N. Miyahara, H. Itoh, M. Endo, and T. Kanai, "Irradiation system for himac," Radiation Research, vol. 48, pp. 15-25, 2007. 
[2] R. B. Hawkins, "A statistical theory of cell killing by radiation of varying linear energy transfer," Radiation Research, vol. 140, pp. 366-374, 1994.

[3] R. B. Hawkins, "A microdosimetric-kinetic model for the effect of non-poisson distribution of lethal lesions on the variation of rbe with let," Radiation Research, vol. 160, pp. 61-69, 2003.

[4] Y. Kase, T. Kannai, Y. Matsumotot, Y. Furusawa, H. Okamato, T. Asaba, M. Sakama, and H. Shinoda, "Mirodosimetric measurements and estimation of human cell survival for heavy-ion beams," Radiation Research Society, vol. 166, pp. 629-638, 2006.

[5] P. D. Bradley, A. B. Rosenfeld, and M. Zaider, "Solid state microdosimetry," Nuclear Instruments and Methods in Physics Research B, vol. 184, pp. 135-157, 2001.

[6] S. Guatelli, M. I. Reinhard, B. Mascialino, D. A. Prokopovich, A. S. Dzurak, M. Zaider, and A. B. Rosenfeld, "Tissue equivalence correction in silicon microdosimetry for protons characteristic of the leo space environment," IEEE Transaction on Nuclear Science, vol. 55, pp. 3407-3413, 2008.

[7] L. T. Tran, S. Guatelli, D. A. Prokopovich, M. Petasecca, M. L. F. Lerch, A. Kok, A. Summanwar, T.E. Hansen, C. D. Via, M. I. Reinhard, J. F. Ziegler, and A. B. Rosenfeld, "3d radiation detectors: Charge collection characterisation and applicability of technology for microdosimetry," IEEE Transaction on Nuclear Science, vol. 61, no. 4, pp. 1537-1543, 2014.

[8] "ICRU Report 36-Microdosimetry," International Commission on Radiation Units and Measurements, 1970.

[9] S. Agostinelli et al., "Geant4 - a simulation toolkit," Nuclear Instruments and Methods in Physics Research Section A: Accelerators, Spectrometers, Detectors and Associated Equipment, vol. 506, no. 3, pp. $250-$ 303, 2003.

[10] J. Allison et al., "Geant4 developments and applications," IEEE Transactions on Nuclear Science, vol. 53, pp. 270-278, Feb 2006.

[11] J. Allison et al., "Recent developments in geant4," Nuclear Instruments and Methods in Physics Research Section A: Accelerators, Spectrometers, Detectors and Associated Equipment, vol. 835, pp. 186 - 225, 2016.

[12] D. Schardt, T. Elsässer, and D. Schulz-Ertner, "Heavy-ion tumor therapy: Physical and radiobiological benefits," Rev. Mod. Phys., vol. 82, pp. 383-425, Feb 2010.

[13] http://physics.nist.gov/PhysRefData/Star/Text/ESTAR.html.

[14] J. A. Davis, S. Guatelli, M. Petasecca, M. L. F. Lerch, M. I. Reinhard, M. Zaider, J. Ziegler, and A. B. Rosenfeld, "Tissue equivalence study of a novel diamond-based microdosimeter for galactic cosmic rays and solar particle events," IEEE Transaction on Nuclear Science, vol. 61, pp. 1544 - 1551, 2014.

[15] L. Jones, P. Hoban, and P. Metcalfe, "The use of the linear quadratic model in radiotherapy: a review," Australasian Physics $\mathcal{E}$ Engineering Sciences in Medicine, vol. 24, no. 3, pp. 132-146, 2001.

[16] http://http://www.nist.gov/pml/data/xraycoef/. 